\section{Cirugía estética de los genitales masculinos}

\author{
Carrillo-Córdova LD, ${ }^{1}$ Carrillo-Esper $\mathrm{R},{ }^{2}$ Carrillo-Córdova JR, ${ }^{3}$ Carrillo-Córdova \\ $\mathrm{CA}^{3}$
}

\section{Resumen}

El tamaño del pene mantiene una relación estrecha con la autoestima e identidad sexual y tiene gran repercusión social, según la idiosincrasia mexicana. La cirugía estética de los genitales masculinos se encuentra en auge desde la década de 1970, por la necesidad de corregir las deficiencias y deformidades físicas que causan estrés psicológico en el hombre. Es importante considerar la valoración preoperatoria y selección de los pacientes para lograr los resultados estéticos deseados. Esta revisión expone la evaluación preoperatoria y las distintas técnicas desarrolladas en el campo de la cirugía estética de los genitales masculinos.

PALABRAS CLAVE: alargamiento de pene, cirugía estética de genitales, engrosamiento del pene.

Rev Mex Urol. 2017 Jul-Aug;77(4):318-327.

\section{Cosmetic surgery of the male genitals}

\author{
Carrillo-Córdova LD, ${ }^{1}$ Carrillo-Esper $\mathrm{R},{ }^{2}$ Carrillo-Córdova JR, ${ }^{3}$ Carrillo-Córdova \\ $\mathrm{CA}^{3}$
}

\begin{abstract}
Penis size is closely related to self-esteem and sexual identity and has a strong social impact in the Mexican culture. Male genital cosmetic surgery has been on the rise since the 1970s, based on the necessity to correct deficiencies and physical deformities that cause significant psychologic stress. It is important to pay special attention to the preoperative evaluation of patients and their selection to achieve the desired results. The present review covers the preoperative preparation and different techniques developed in the field of male cosmetic surgery.

KEYWORDS: Penile lengthening; Genital cosmetic surgery; Penile girth enhancement
\end{abstract}

${ }^{1}$ Departamento de Urología, Hospital General de México Dr. Eduardo Liceaga, Ciudad de México. ${ }^{2}$ Jefe de la División de Áreas Críticas (CENIAQ), Instituto Nacional de Rehabilitación, Ciudad de México. ${ }^{3}$ Departamento de Cirugía Plástica y Reconstructiva, Hospital General Dr. Manuel Gea González, Ciudad de México.

Recibido: diciembre 2016

Aceptado: junio 2017

Correspondencia

Dr. Luis Daniel Carrillo Córdova

Carrillocor@gmail.com

Este artículo debe citarse como

Carrillo-Córdova LD, Carrillo-Esper R, CarrilloCórdova JR, Carrillo-Córdova CA. Cirugía estética de los genitales masculinos. Rev Mex Urol. 2017 jul-agos;77(4):318-327.

DOI: https://doi.org/10.24245/revmexurol.v77i4.1119 


\section{ANTECEDENTES}

La percepción de algunos pacientes hacia sus genitales tiene función importante en su identidad sexual, autoestima y calidad de vida. En la idiosincrasia mexicana, el tamaño y grosor del pene se relacionan, de manera popular, con la virilidad y potencia sexual. Debido a la gran popularidad del internet y con ello el fácil acceso a la pornografía, se muestra un patrón falso del tamaño normal de los genitales masculinos, ya que suelen exponerlos de gran tamaño y grosor.

En años recientes, la cirugía de aumento de tamaño del pene se ha convertido en una práctica relativamente común, sobre todo en el medio privado. Aun así, este procedimiento no se encuentra estandarizado, lo que lleva a un gran número de intervenciones con resultados pobremente documentados. Desde el inicio de la década de 1970 los medios han contribuido a la popularidad de estos procedimientos; como consecuencia, en Estados Unidos se registró que 10,000 hombres se realizaron cirugías de aumento de pene entre 1991 y 1998. La mayoría de esos pacientes buscó atención médica secundaria a la percepción del tamaño pequeño de su pene en estado de flacidez, o comúnmente Ilamado "síndrome del vestidor ${ }^{\prime \prime}{ }^{1-3}$

La mayoría de los pacientes que solicita cirugía estética tiene genitales normales en cuanto a longitud y grosor, según nomogramas regionales (Cuadro 1). En Estados Unidos se ha reportado que el promedio normal del pene en erección es de $14-15 \mathrm{~cm}$ y la circunferencia de $12.2 \mathrm{~cm} .{ }^{4}$ En una serie de 3300 adultos jóvenes italianos se encontró que la longitud promedio en flacidez fue de $9 \mathrm{~cm}$ y circunferencia de $10 \mathrm{~cm} .{ }^{5}$ Otro estudio en la población hindú, efectuado en 301 pacientes, reportó una longitud en flacidez de $8.2 \mathrm{~cm}$ y circunferencia de $9.14 \mathrm{~cm}$, y en erección una longitud de $13 \mathrm{~cm}$ y circunferencia de $11.46 \mathrm{~cm} .{ }^{6}$ La revisión sistemática más grande publicada hasta la fecha incluyó 15,521 hombres y reportó los siguientes valores: en estado de flacidez una longitud promedio de $9.16 \mathrm{~cm}$ y en erección de $13.12 \mathrm{~cm}$, y la circunferencia en reposo de $9.31 \mathrm{~cm}$ y en erección de $11.66 \mathrm{~cm}^{7}$

En un estudio italiano, 44 (65.7\%) adultos jóvenes se quejaron del pene corto flácido, 22 $(32.8 \%)$ de su miembro flácido y erecto, y sólo $1(1.5 \%)$ estaba preocupado por la longitud erecta del pene; además, 15 (22.4\%) sujetos expresaron inconformidad con la circunferencia; 57 (85\%) pensaron que la longitud normal del pene debería ser de 10 a $17 \mathrm{~cm}$ (mediana de $12 \mathrm{~cm})$ y $10(15 \%)$ casos fueron incapaces de estimar el tamaño del pene "normal". No reportaron ningún caso con longitud del pene con percentil de 2.5, según su nomograma. Cuarenta y dos $(62.7 \%)$ sujetos mencionaron su problema desde la infancia, cuando sentían que su pene era más pequeño que el de sus amigos. En 25 (37.3\%) casos el problema comenzó en la adolescencia, después de observar imágenes eróticas. Concluyeron que la mayoría de los hombres que buscan la cirugía de alargamiento del pene sobrestiman la longitud "normal" de su miembro. En esa serie, ninguno de los pacientes pudo clasificarse con pene severamente corto, de acuerdo con su nomograma, ni tenían anomalías anatómicas del miembro. ${ }^{8}$

Hoy en día los urólogos y cirujanos plásticos cuentan con técnicas quirúrgicas bien establecidas que permiten mejorar la apariencia estética, longitud y grosor del pene y escroto; sin embargo, siempre deben estudiarse los motivos del paciente para someterlo a este tipo de procedimientos, además de efectuar una evaluación psiquiátrica previa a cualquier intervención y ofrecer una explicación clara de los resultados reales que se espera encontrar. 
Cuadro 1. Medidas (longitud y circunferencia) del pene, según los resultados obtenidos en distintas series

\begin{tabular}{lcc|c|c|c|c|c|c} 
Autor & Año & $\mathbf{n}$ & Edad & $\begin{array}{c}\text { Longitud } \\
\text { (flácido) }\end{array}$ & $\begin{array}{c}\text { Longitud } \\
\text { (estirado) }\end{array}$ & $\begin{array}{c}\text { Longitud } \\
\text { (erecto) }\end{array}$ & $\begin{array}{c}\text { Circunferencia } \\
\text { (flácido) }\end{array}$ & $\begin{array}{c}\text { Circunferencia } \\
\text { (erecto) }\end{array}$ \\
\hline Loeb & 1899 & 50 & $17-35$ & 9.41 & - & - & - & - \\
\hline Schonfeld & 1942 & 196 & $17-25$ & - & 13.1 & - & 8.5 & 15.8 \\
\hline Kinsey & 1948 & 2770 & $20-59$ & 9.7 & 16.74 & - & - & - \\
\hline Bondil & 1992 & 905 & $17-91$ & 10.7 & 16.74 & - & - & - \\
\hline De Ros & 1994 & 150 & - & - & - & 14.5 & 11.5 & - \\
Wessells & 1996 & 80 & $21-82$ & 8.85 & 12.45 & 12.89 & 9.71 & 12.30 \\
\hline Bogaert & 1999 & 935 & - & 10.41 & - & 16.4 & 9.75 & 12.57 \\
\hline Ponchietti & 2001 & 3300 & $17-19$ & 9 & 12.5 & - & 10 & - \\
\hline Schneider & 2001 & 111 & $18-32$ & 8.6 & - & 14.48 & - & - \\
\hline Awwad & 2005 & 271 & $17-83$ & 9.3 & 13.5 & - & 8.9 & - \\
Herbenick & 2015 & 365 & - & - & - & 14.15 & - & 12.23 \\
Veale & 2015 & 15521 & - & 9.16 & 13.24 & 13.2 & 9 & 11.66
\end{tabular}

\section{Evaluación preoperatoria}

Para seleccionar un paciente a cirugía, es necesario el tratamiento multidisciplinario y realizar: historia clínica, examen físico, determinaciones séricas hormonales; evaluación psiquiátrica y psicosexual completas y aplicación del cuestionario APPSSI (Augmentation Phalloplasty Patient Selection and Satisfaction Inventory) (Cuadro 2). ${ }^{9,10}$

Antes de realizar cualquier procedimiento de faloplastia, de aumento en genitales, es importante discutir y documentar las metas que busca obtener el paciente. Se han desarrollado diversos métodos para la selección de estos pacientes y entre los más estudiados se encuentra la aplicación del cuestionario APPSSI. Su finalidad es realizar una evaluación cuantitativa de la severidad del padecimiento y el deseo del paciente a someterse a una cirugía de alargamiento de pene, además de realizar una evaluación numérica relacionada con el resultado posquirúrgico. ${ }^{10}$

El APPSSI consta de cuatro preguntas y cada una puede contestarse con cinco respuestas posibles, con base en un valor numérico (escala de 0-4). Las preguntas 1 y 3 se responden de manera preoperatoria (evaluación de la idoneidad) y las preguntas 1, 2 y 4 de forma postoperatoria (evaluación de los resultados). El umbral de elegibilidad de la cirugía se realiza con base en una puntuación de 6 o menor. Los puntajes preoperatorios varían de 0 (cirugía justificada) a 12 (cirugía no justificada), al igual que los posoperatorios: 0 (decepcionado) y 12 (muy contento o satisfecho). Se necesitan series más grandes, con mayor número de pacientes, para validar el cuestionario. ${ }^{10}$

Deben efectuarse mediciones de la longitud y circunferencia del pene de manera preoperatoria. Medirlo en estado de flacidez, desde la unión pubo-peneana a la punta del glande, en la cara dorsal del pene, y repetirla traccionando el glande (medición del pene flácido y flácido con tracción). La circunferencia se mide de la base del pene al surco balanoprepucial. ${ }^{9}$

Aún se discute si el alargamiento o engrosamiento del pene es un procedimiento ético en pacientes con genitales de tamaño normal. Uno 
Cuadro 2. Cuestionario APPSSI (Augmentation Phalloplasty Patient Selection And Satisfaction Inventory) traducido al español

\begin{tabular}{|c|c|c|c|c|c|}
\hline Muy bajo & Bajo & $\begin{array}{l}\text { Bastante } \\
\text { alterado, pero } \\
\text { suficiente }\end{array}$ & Alto & Muy Alto & $\begin{array}{l}\text { ¿Cómo calificaría su auto- } \\
\text { estima en relación con la } \\
\text { percepción de su imagen } \\
\text { corporal, respecto del ta- } \\
\text { maño de su pene? }\end{array}$ \\
\hline $\begin{array}{c}0 \\
\text { Muy bajo }\end{array}$ & $\begin{array}{c}1 \\
\text { bajo }\end{array}$ & $\begin{array}{c}2 \\
\text { Bastante } \\
\text { alterado, pero } \\
\text { suficiente }\end{array}$ & $\begin{array}{c}3 \\
\text { Alto }\end{array}$ & $\begin{array}{c}4 \\
\text { Muy Alto }\end{array}$ & $\begin{array}{l}\text { ¿Cómo calificaría su au- } \\
\text { toestima al intentar tener } \\
\text { relaciones sexuales con } \\
\text { una persona, respecto del } \\
\text { tamaño de su pene? }\end{array}$ \\
\hline $\begin{array}{c}\mathbf{0} \\
\text { Extremadamente } \\
\text { significante } \\
\text { Considero este } \\
\text { procedimiento } \\
\text { extremadamente } \\
\text { necesario para } \\
\text { mi, sin importar } \\
\text { los resultados }\end{array}$ & $\begin{array}{c}1 \\
\text { Muy Significante } \\
\text { Es mi decisión } \\
\text { someterme a } \\
\text { este procedi- } \\
\text { miento, esperan- } \\
\text { do el mejor de } \\
\text { los resultados }\end{array}$ & $\begin{array}{c}2 \\
\text { Moderadamente } \\
\text { significante } \\
\text { Deseo some- } \\
\text { terme a este } \\
\text { procedimiento, } \\
\text { pero me gustaría } \\
\text { pensarlo más a } \\
\text { fondo }\end{array}$ & $\begin{array}{c}\mathbf{3} \\
\text { Poco significante } \\
\text { Me gustaría so- } \\
\text { meterme a este } \\
\text { procedimiento si } \\
\text { garantiza un re- } \\
\text { sultado positivo }\end{array}$ & $\begin{array}{c}\mathbf{4} \\
\text { Insignificante } \\
\text { Nunca conside- } \\
\text { raría esta opción }\end{array}$ & $\begin{array}{l}\text { ¿Qué tan importante consi- } \\
\text { dera la opción de someterse } \\
\text { a un procedimiento de falo- } \\
\text { plastia de aumento para al- } \\
\text { terar su aspecto después de } \\
\text { informarse a fondo respecto } \\
\text { de la medida objetiva de su } \\
\text { pene, las expectativas reales } \\
\text { y posibles complicaciones } \\
\text { de la operación? }\end{array}$ \\
\hline $\begin{array}{c}\mathbf{0} \\
\text { Decepcionado } \\
\text { Mi condición } \\
\text { es peor que la } \\
\text { preoperatoria }\end{array}$ & $\begin{array}{c}1 \\
\text { Insatisfecho } \\
\text { No hay diferen- } \\
\text { cias a pesar de } \\
\text { las molestias }\end{array}$ & $\begin{array}{c}2 \\
\text { Indiferente } \\
\text { Muy poca } \\
\text { mejoría }\end{array}$ & $\begin{array}{c}3 \\
\text { Satisfecho } \\
\text { Mejoría signifi- } \\
\text { cativa, valió la } \\
\text { pena someter- } \\
\text { me a todos los } \\
\text { problemas del } \\
\text { procedimiento }\end{array}$ & $\begin{array}{c}4 \\
\text { Muy contento } \\
\text { o satisfecho } \\
\text { El resultado es } \\
\text { mucho mejor de } \\
\text { lo esperado }\end{array}$ & $\begin{array}{l}\text { Puntaje total (Q1-2-3) } \\
\text { ¿Cómo se siente con el } \\
\text { resultado final de la cirugía, } \\
\text { en relación con su condi- } \\
\text { ción preoperatoria y sus } \\
\text { expectativas? }\end{array}$ \\
\hline $\begin{array}{c}0 \\
\text { Puntaje (1-2-4) }\end{array}$ & $\begin{array}{c}1 \\
\text { Puntaje (1-2-4) }\end{array}$ & $\begin{array}{c}2 \\
\text { Puntaje (1-2-4) }\end{array}$ & $\begin{array}{c}3 \\
\text { Puntaje (1-2-4) }\end{array}$ & $\begin{array}{c}4 \\
\text { Puntaje (1-2-4) }\end{array}$ & $\begin{array}{l}\text { Puntaje Total } \\
\text { (P 1-2-4) }\end{array}$ \\
\hline
\end{tabular}

de los primeros trabajos en abordar este tema fue el de Chi-Ying Li y su grupo, quienes evaluaron de manera objetiva la satisfacción posoperatoria de pacientes intervenidos de alargamiento del pene, mediante la incisión del ligamento suspensorio del mismo. La conclusión más importante fue que los pacientes con tamaño normal del pene deben buscar apoyo psicosexual y los médicos desaconsejar el procedimiento. ${ }^{11}$

Hasta el momento no existe información contundente de las complicaciones relacionadas con la cirugía de alargamiento o ensanchamiento del pene. La incisión del ligamento suspensorio pue- de causar un menor ángulo durante la erección y se han reportado algunos casos de acortamiento paroxístico después del procedimiento. ${ }^{12,13} \mathrm{Un}$ colgajo de avance puede provocar deformación severa del pene, con cicatrización deficiente y formación de heridas hipertróficas. ${ }^{14}$

Desde el punto de vista quirúrgico, las técnicas actuales no se han investigado científicamente. Diversos factores están implicados en la evaluación de estos procedimientos, por ejemplo: ¿Es posible alargar o ensanchar el pene de estos pacientes?, se ha demostrado que los distintos procedimientos solo aumentan la longitud $1 \mathrm{~cm}$; 
por tanto, ¿qué pacientes desean someterse a este tipo de procedimiento?, ¿la ansiedad y depresión de los pacientes se relaciona con su decisión de someterse a este tipo de procedimientos? y ilos grados de ansiedad y depresión disminuyen después de la intervención quirúrgica? ${ }^{1,15}$

La cirugía de alargamiento o ensanchamiento del pene, en caso de ser necesaria, sigue siendo un procedimiento controvertido y debe considerarse tema de investigación; por tanto, debe ofrecerse la opción de procedimientos no quirúrgicos, menos invasivos, y recurrir como último recurso a los colgajos. Actualmente, la mayoría se encontrará insatisfecho después del procedimiento. Se necesitan más ensayos clínicos aleatorizados y la creación de instrumentos validados que permitan la adecuada selección de este tipo de pacientes. ${ }^{15}$

\section{Condiciones asociadas con el acortamiento del pene}

Existen alteraciones, médicas o quirúrgicas, asociadas con el acortamiento del pene, por ejemplo: pacientes con cáncer de próstata tratados mediante prostatectomía radical (68-71\% pierden, en promedio, $1 \mathrm{~cm}$ de longitud), sujetos que reciben bloqueo androgénico total con radioterapia (longitud prequirúrgica del pene en estado flácido menor de $14 \mathrm{~cm}$ ), pacientes sometidos únicamente a radioterapia; enfermedad de Peyronie y desórdenes congénitos; además, se ha demostrado acortamiento del pene en pacientes con disfunción eréctil. ${ }^{16-22}$

\section{Alargamiento del pene}

Hoy en día existen diversas técnicas quirúrgicas para alargamiento del pene; una de las más practicadas es la liberación del ligamento suspensorio del pene, ${ }^{13}$ en la que se libera el ancho de un dedo a través de la longitud de la sínfisis del pubis, incidiendo directamente en el periostio, a través de su línea media, para lograr el avance de los cuerpos cavernosos. La intervención suele efectuarse mediante una incisión transversal, por encima de la unión pene-púbica, y después se aplica tracción sobre el pene, utilizando diversos métodos, ya sea succión con vacío, pesas o equipos especializados. ${ }^{1}$

Con este procedimiento se aumentan de 1 a 2 $\mathrm{cm}$, aunque algunas series reportan que no existe ganancia. Una de las medidas relacionadas con mayor éxito en el alargamiento del pene ha sido la colocación de pesarios o sistemas de tracción posoperatorios, que pueden usarse una semana después del procedimiento y continuar entre 6 y 24 meses.

Entre las complicaciones del procedimiento se ha descrito pérdida del ángulo del pene en erección, ${ }^{13}$ encogimiento paroxístico asociado con cicatrización de los cuerpos cavernosos con el ligamento liberado (para prevenirlo se ha recomendado colocar una barra de silicón en el espacio creado por la división del ligamento, incluso la colocación de colgajo de grasa en esta misma zona o un colgajo de escroto o piel que recubra los cuerpos cavernosos en su región proximal). ${ }^{18-20}$ Otra complicación descrita con esta técnica es la inestabilidad del pene, provocada por la disección agresiva del ligamento suspensorio. ${ }^{12}$

Otro procedimiento comúnmente utilizado es el colgajo de avance de piel sobre el pene. El fundamento de esta técnica es hacer que la porción distal del pene protruya por medio de un colgajo o una plastia $\mathrm{V}-\mathrm{Y}$ realizada en la base del mismo. Entre las diferentes técnicas descritas de colgajos de avance, los mejores resultados se obtienen con la técnica de $Z$ plastia doble. ${ }^{12}$

El colgajo de avance $\mathrm{V}$-Y realizado en la unión pene-púbica puede ofrecer una apariencia de 
mayor longitud del pene flácido; sin embargo, esta técnica se asocia con complicaciones como: interrupción del flujo sanguíneo normal del pene, que provoca problemas de cicatrización de la herida, pérdida total o parcial del colgajo y dehiscencia. Es común la formación de cicatrices amplias, lo que provoca pérdida de vello púbico y hundamientos en el pubis. ${ }^{12,23}$

El colgajo de avance $\mathrm{V}-\mathrm{Y}$ resulta en un colgajo grueso de piel, que puede originar abultamiento poco estético y crecimiento de vello en la base del pene. ${ }^{23}$

Otro procedimiento para alargar el pene es el uso no invasivo de pesarios o equipos diseñados especialmente para provocar la tracción permanente del miembro. Estas técnicas requieren que el paciente las utilice de 4 a 9 horas diarias, entre 3 y 6 meses, con lo que se obtiene un promedio de alargamiento de 2.3 a $2.7 \mathrm{~cm} .{ }^{24,25}$

\section{Engrosamiento del pene}

El engrosamiento del pene es una técnica aún más debatida que el alargamiento, pues hasta la fecha no existe ninguna indicación absoluta para realizar este procedimiento. La única guía realizada para el alargamiento del pene señala que no existe fundamento para engrosarlo. ${ }^{26}$

Con todo esto, la mayoría de los pacientes que buscan alargamiento del pene, también desean el engrosamiento. La clave de estos procedimientos es lograr un cilindro simétrico, con circunferencia aumentada en todos sus aspectos, que es muy difícil de lograr. Estas intervenciones suelen generar complicaciones y deformidades.

El engrosamiento puede realizarse con la colocación subcutánea de distintos tejidos (grasa, injerto de dermis, etc.) o mediante aumento cavernoso con injertos de vena safena. ${ }^{14}$
La inyección autóloga de grasa en la fascia de dartos es uno de los procedimientos más utilizados para lograr el engrosamiento del pene. Entre sus ventajas se reporta que no existe rechazo y el material tiene buena disponibilidad. Los resultados de esta técnica dependen de la experiencia del cirujano. La inyección de pequeñas cantidades de grasa, mediante diversos túneles, se asocia con bajo índice de deformidad. Entre sus desventajas, representa un alto grado de reabsorción. Un estudio demostró que después de 1 año solo permanece $30 \%$ de la grasa inyectada. ${ }^{27}$ Cuando la intervención se realiza inadecuadamente o se inyectan grandes cantidades de grasa, pueden formarse nódulos, una superficie irregular, asimetría del pene o falta de rigidez por exceso de grasa. Sin embargo, se ha descrito que la circunferencia aumenta de 2 a $3 \mathrm{~cm}$ con esta técnica. ${ }^{28}$

En 2006, Panfilov y su grupo describieron un método para inyectar grasa autóloga en el pene. En su estudio aplicaron $200-250 \mathrm{~cm}^{3}$ de solución fisiológica con adrenalina (1:800 00) y lidocaína al $0.02 \%$ (50 mL de lidocaína al $1 \%$ por $1 \mathrm{~mL}$ de solución), y realizaron dos incisiones de 2 a 3 mm en el muslo interno para inyectar la solución; después de 50 minutos recolectaron la grasa, que se inyectó en el pene mediante 4 incisiones de $1 \mathrm{~mm}$ a la 1, 5, 7 y 11, según la caratula del reloj. En cada incisión se colocaron de 10 a 16 $\mathrm{cm}^{3}$ de grasa, posteriormente se dio un masaje para distribuir la grasa uniformemente. Después de un año de seguimiento, 77 pacientes estaban satisfechos, 8 moderadamente y 3 insatisfechos. ${ }^{29}$

Otra técnica descrita es la colocación de injertos de dermis, en forma de láminas entre la fascia de dartos, incluso puede colocarse un injerto amplio, que cubra al dartos en toda su circunferencia. El resultado estético es excelente, con una superficie uniforme y lisa; sin embargo, es un procedimiento quirúrgico prolongado y se ha reportado edema del pene 
durante 6 semanas. La textura normal del pene se logra entre el cuarto y sexto mes posquirúrgico, y en promedio la circunferencia aumenta entre 2 y $4 \mathrm{~cm}^{28}$

La colocación de injertos puede provocar complicaciones importantes si no logra acoplarse por completo; por ejemplo: necrosis, formación de cicatrices, acortamiento del pene y curvaturas patológicas.

El injerto AlloDerm ${ }^{\circledR}$ para engrosamiento del pene se coloca en capas sobre la fascia de Buck y se sutura de manera proximal y distal. Entre las complicaciones se han descrito deformidades, asimetría, acortamiento del pene, formación de curvaturas patológicas e infección de la herida quirúrgica. ${ }^{28}$

El uso de materiales sintéticos incluye: inyección de silicón líquido (proporciona buenos resultados a corto plazo, pero a futuro provoca diversos tipos de deformidades y formación de nódulos) e inyección de ácido hialurónico en el glande y la fascia de Buck (ha demostrado buenos resultados; sin embargo, hasta el momento no se han reportado resultados a largo plazo). ${ }^{30}$

Otra técnica incluye colocar un injerto de vena safena en los cuerpos cavernosos, cuyo procedimiento se inicia con una incisión en la túnica albugínea, del ápex a la base, y se coloca un parche de vena safena. ${ }^{14}$ Esta técnica no ha mostrado diferencia significativa en el diámetro del pene flácido, pero la circunferencia en erección aumenta significativamente $(2.85 \mathrm{~cm}$ en el preoperatorio versus $4.1 \mathrm{~cm}$ en el posoperatorio). Por el momento no ha reportado complicaciones. ${ }^{14}$

Con el advenimiento de la bioingeniería se han desarrollado nuevos materiales para aumentar el diámetro del pene. Perovic y sus coautores describieron un material biodegradable utili- zado en 84 pacientes con esta finalidad. Se cultivan fibroblastos de piel del escroto hasta obtener un volumen de células de $2 \times 10^{7}$ y posteriormente se colocan en una matriz tubular de ácido polilacticoglicólico; después de 24 horas de incubación, se realiza el desforramiento del pene y la matriz se coloca entre la fascia de dartos y Buck. En el seguimiento a los 24 meses se observó aumento de la circunferencia del pene de $3.15 \mathrm{~cm}(1.9 \mathrm{a} 4.1 \mathrm{~cm})$ en estado de flacidez y de $2.47 \mathrm{~cm}(1.8-3.0)$ en erección. ${ }^{31} \mathrm{El}$ Cuadro 3 muestra los resultados de diferentes estudios.

\section{Pene oculto}

Este término se refiere a cualquier alteración o percepción en la que el pene se vea "disminuido" de tamaño, secundario a la acumulación de grasa peripeneana, suprapúbica o escrotal. La mayoría de los pacientes tiene longitud peneana normal, pero se altera por ciertas condiciones. El tratamiento de esta alteración tiene como finalidad disminuir la grasa suprapúbica y, en algunos casos, la resección de piel. ${ }^{32}$

1. Liposucción suprapúbica: este procedimiento se indica en pacientes con mínima laxitud de piel, cuyo contenido de grasa suprapúbica es considerable. Debe tenerse extrema precaución con este método, debido a la alta vascularidad de la región.

Cuadro 3. Ganancia en cm para engrosamiento del pene, según distintas publicaciones

\begin{tabular}{l|c|c|c|c|c|} 
Autor & Año & $\mathbf{n}$ & Edad & $\begin{array}{c}\text { Ganancia } \\
\text { en } \mathbf{~ c m} \\
\text { (flácido) }\end{array}$ & $\begin{array}{c}\text { Ganancia } \\
\text { en } \mathbf{~ c m} \\
\text { (erecto) }\end{array}$ \\
$\begin{array}{l}\text { Austoni y } \\
\text { col. }\end{array}$ & 2002 & 39 & $24-47$ & 0.15 & 1.36 \\
$\begin{array}{l}\text { Perovic y } \\
\text { col. }\end{array}$ & 2006 & 204 & $19-54$ & 3.15 & 2.47 \\
$\begin{array}{l}\text { Panfilovy } \\
\text { col. }\end{array}$ & 2006 & 60 & $24-34$ & 2.65 & - \\
\hline
\end{tabular}


Las complicaciones más frecuentes son seroma y hematoma. ${ }^{33}$

2. Resección suprapúbica de piel y tejido celular subcutáneo: esta intervención suele acompañarse de liposucción y se indica en pacientes con exceso de piel posterior a la liposucción. La resección de piel y tejido celular subcutáneo no es un procedimiento sencillo, debe realizarse plicatura de la piel subcutánea, hasta la fascia del recto abdominal, para evitar telescopaje del pene. ${ }^{34}$

Con ambos procedimientos puede corregirse la mayor parte de los padecimientos relacionados con el pene oculto. Es importante considerar una etapa posoperatoria de reposo y vigilancia, debido a la posibilidad de hematomas o seromas.

\section{Reducción escrotal}

El aumento de piel del escroto se relaciona con inconformidad psicosocial, mala salud sexual, irritación cutánea e incomodidad para realizar actividad física. Las causas relacionadas con esta alteración comprenden: edad avanzada, secuelas de hidrocele o varicocele, obesidad o laxitud congénita de la piel escrotal. El tratamiento consiste en cirugía en todos los casos. Debe considerarse preservar el escroto posterior, debido a su alta concentración de drenaje linfático. Entre las cirugías de escroto se encuentran:

1. Resección en huso: está indicada en pacientes con mínima-media laxitud de la piel escrotal. Debe considerarse el rafé escrotal como punto central, incidir la piel y el tejido celular hasta la fascia de dartos. Posteriormente se cierra por planos, con puntos absorbibles, la fascia de dartos y enseguida el tejido celular subcutáneo. La piel puede cerrarse con suturas absorbibles de larga duración, para evitar el malestar de retirar puntos en esta zona.
2. Z-plastia: es el método de elección en resección de piel de manera asimétrica o cuando se planea resecar cantidades medias-grandes de piel escrotal. Con esta técnica se pretende disminuir la tensión de la herida y la cicatriz posquirúrgica. El cierre se realiza de la misma manera que en la resección en huso.

3. Corrección del pliegue pene-escrotal: esta técnica genera problemas durante la erección e inconformidad estética, debido a la inserción alta del escroto, a nivel del pene. El objetivo del tratamiento es retirar el exceso de piel, con la mínima cicatriz posible para evitar problemas funcionales posteriores. Es importante planear la cirugía y considerar la Z-plastia para eliminar la cicatriz que pueda formarse a futuro. ${ }^{35} \mathrm{En}$ algunos casos puede realizarse un avance $\mathrm{V}-\mathrm{Y}$, con el que se lleva el exceso de piel hacia atrás. Algunas series pequeñas de casos demuestran buenos resultados con la escrotoplastia en doble $\mathrm{V}^{36}$

Este tipo de procedimientos deben realizarse en el quirófano y tener extremo cuidado en la hemostasia, debido a que los hematomas o seromas son la primera complicación reportada, incluso pueden provocar dehiscencia de la herida..$^{37,38}$

\section{CONCLUSIÓN}

La cirugía estética de los genitales masculinos se ha convertido en un procedimiento de gran popularidad. El conocimiento detallado de la anatomía y las técnicas quirúrgicas es fundamental para el éxito de estos procedimientos. Las intervenciones más frecuentemente realizadas van encaminadas hacia el alargamiento y engrosamiento del cuerpo del pene. Los procedimientos relacionados con la grasa suprapúbica y la bolsa escrotal se practican cada vez con mayor frecuencia. Las complicaciones más comunes 
son infecciones, seromas y hematomas; por tanto, la hemostasia transoperatoria y la higiene son factores fundamentales al momento de realizar este tipo de procedimientos complejos.

\section{REFERENCIAS}

1. Hinman F. Microphallus: characteristics and choice of treatment from a study of 20 cases. J Urol 1972;107:499505.

2. Kelley JH, Eraklis AJ. A procedure for lengthening the phallus in boys with extrophy of the bladder. J Pediatr Surg 1971;6:645-9.

3. Van Driel MF, Schultz WC, Van de Wiel HB, Mensink HJ. Surgical lengthening of the penis. Br J Urol 1998;82:81-5.

4. Herbenick D, Reece M, Schick V, Sanders SA. Erect penile length and circumference dimensions of 1,661 sexually active men in the United States. J Sex Med 2014;11:93-101.

5. Ponchietti R, Mondaini N, Bonafè $M$, Di Loro F, Biscioni $S$, Masieri L. Penile length and circumference: a study on 3,300 young Italian males. Eur Urol. 2001;39(2):183-6.

6. Promodu K, Shanmughadas KV, Bhat S. Penile length and circumference: an Indian study. Int J Impot Res. 2007:19(6);558-63.

7. Veale D, Miles S, Bramley S, Muir G, Hodsoll J. Am I normal? A systematic review and construction of nomograms for flaccid and erect penis length and circumference in up to 15,521 men. BJU Int. 2015;115(6):978-86. doi: 10.1111/ bju.13010.

8. Mondaini, R Ponchietti, P Gontero, GH Muir, A Natal, F Di Loro, et al. Penile length is normal in most men seeking penile lengthening procedures. Int J Imp Res. 2002:14;283-286.

9. Spyropoulosa E, Christoforidisb C, Borousasa D, Mavrikosa $S$, Bourounisa $M$, Athanasiadis $S$. Augmentation phalloplasty surgery for penile dysmorphophobia in young adults: considerations regarding patient selection, outcome evaluation and techniques applied. Eur Urol. 2005:48(1);121-128.

10. Spyropoulos E, Galanakis I, Dellis A. Augmentation PhaIloplasty Patient Selection and Satisfaction Inventory: a novel questionnaire to evaluate patients considered for augmentation phalloplasty surgery because of penile dysmorphophobia. Urology. 2007; 70(2):221-6.

11. Li CY, Kayes O, Kell PD, Christopher N, Minhas S, Ralph DJ. Penile suspensory ligament division for penile augmentation: indications and results. Eur Urol 2006;49:729-33.

12. Wessells H, Lue TF, McAninch JW. Complications of penile lengthening and augmentation seen at 1 referral center. J Urol 1996;156:1617-20.

13. Alter GJ. Augmentation phalloplasty. Urol Clin North Am 1995;22:887-902.
14. Austoni E, Guarneri A, Cazzaniga A. A new technique for augmentation phalloplasty: albugineal surgery with bilateral saphenous grafts - three years of experience. Eur Urol 2002;42:245-53.

15. Vardi $Y$. Is penile enlargement an ethical procedure for patients with a normal-sized penis? Eur Urol. 2006 ;49(4):609-11.

16. Awwad Z, Abu-Hijleh M, Basri S, Shegam N, Murshidi M, Ajlouni K. Penile measurements in normal adult Jordanians and in patients with erectile dysfunction. Int J Impot Res 2005;17:191-195.

17. Munding MD, Wessells HB, Dalkin BL. Pilot study of changes in stretched penile length 3 months after radical retropubic prostatectomy. Urology 2001;58:567-569.

18. Savoie M, Kim SS, Soloway MS. A prospective study measuring penile length in men treated with radical prostatectomy for prostate cancer. J Urol 2003;169:1462-1464.

19. Haliloglu A, Baltaci S, Yaman O. Penile length changes in men treated with androgen suppression plus radiation therapy for local or locally advanced prostate cancer. J Urol 2007;177:128-130.

20. Hall SJ, Basile G, Bertero EB, de las Morenas A, Goldstein I. Extensive corporeal fibrosis after penile irradiation. J Urol 1995;153:372-377.

21. Pryor J, Akkus E, Alter $\mathrm{G}$, Jordan $\mathrm{G}$, Lebret $\mathrm{T}$, Levine $\mathrm{L}$, et al. Peyronie's disease. J Sex Med 2004;1:110-115.

22. Rigaud G, Berger RE. Corrective procedures for penile shortening due to Peyronies's disease. J Urol 1995;153:368-370.

23. Shirong L, Xuan Z, Zhengxiang W, Dongli F, Julong W, Dongyun $Y$. Modified penis lengthening surgery: review of 52 cases. Plast Reconstr Surg 2000;105(2):596-599.

24. Gontero P, Di Marco M, Giubilei G, et al. A pilot phase-II prospective study to test the 'efficacy' and tolerability of a penile-extender device in the treatment of 'short penis'. BJU Int 2009;103(6):793-797.

25. Nikoobakht M, Shahnazari A, Rezaeidanesh M, Mehrsai A, Pourmand G. Effect of penile-extender device in increasing penile size in men with shortened penis: preliminary results. J Sex Med 2011;8(11):3188-92.

26. Wessells H, Lue TF, McAninch JW. Penile length in the flaccid and erect states: guidelines for penile augmenta- tion. $J$ Urol 1996;156:995-7.

27. American Society of Plastic and reconstructive surgeons. Report on autologous fat transplantation by the ASPRS ad hoc committee on new procedures. Chicago: American Society of Plastic and Reconstructive Surgeons (ASPRS); 1987.

28. Alter GJ, Salgado CJ, Chim H. Aesthetic surgery of the male genitalia. Semin Plast Surg 2011;25(3):189-95.

29. Panfilov DE. Augmentative phalloplasty. Aesthetic Plast Surg 2006;30:183-197.

30. Kim JJ, Kwak TI, Jeon BG, Cheon J, Moon DG. Human glans penis augmentation using injectable hyaluronic acid gel. Int J Impot Res 2003;15(6):439-443. 
Carrillo-Córdova LD y col. Cirugía estética de los genitales masculinos

31. Perovic SV, Byun JS, Scheplev P, Djordjevic ML, Kim JH, Bubanj T. New perspectives of penile enhancement surgery: tissue engineering with biodegradable scaffolds. Eur Urol 2006;49:139-147.

32. Chevallier D, Haertig A, Faix A, Droupy S. Cosmetic surgery of the male genitalia. Prog Urol. 2013;23:685-695.

33. Alter GJ. Surgical techniques: surgery to correct hidden penis. J Sex Med 2006;3(5):939-42.

34. Adham MN, Teimourian B, Mosca P. Buried penis release in adults with suction lipectomy and abdominoplasty. Plast Reconstr Surg 2000;106(4):840-844
35. Alter GJ. Correction of penoscrotal web. J Sex Med 2007;4:844-847.

36. McLeod DJ, Alpert SA. Double V scrotoplasty for repair of congenital penoscrotal webbing: A hidden scar technique. J Pediatr Surg 2014; 10:810-814.

37. Alter GJ. Aesthetic genital surgery. In: Mathes SJ, Hentz VR, eds. Plastic Surgery. $2^{\text {nd }}$ ed. Philadelphia: WB Saunders, 2000;389-410.

38. Machov JA, Langenstroer $P$, Sanger JR. Surgical reduction of massive localized lymphedema (MLL) in obesity. JPRAS 2014;67:1719-1725.

\section{AVISO PARA LOS AUTORES}

Revista Mexicana de Urología tiene una nueva plataforma de gestión para envío de artículos: https://www.revisionporpares.com/index.php/RMUrol ahí podrá inscribirse a la base de datos administrada por el sistema Open Journal System (OJS) que ofrece las siguientes ventajas para los autores:

- Subir sus artículos directamente al sistema.

- Conocer, en cualquier momento, el estado de los artículos enviados, es decir, si ya fueron asignados a un revisor, aceptados con o sin cambios, o rechazados.

- Participar en el proceso editorial corrigiendo y modificando sus artículos hasta su aceptación final. 